\title{
NOVEL METHOD TO FIND THE PARAMETER FOR NOISE REMOVAL FROM MULTI-CHANNEL ECG WAVEFORMS
}

\author{
Menta Srinivasulu', K. Chennakeshava Reddy ${ }^{2}$ \\ ${ }^{1}$ Professor and HOD ECE Dept. LITS, Khammam, AP, India \\ ${ }^{2}$ Principal, BIET, Ibrahimpatnam, AP, India
}

\begin{abstract}
In general, electrocardiogram (ECG) waveforms are affected by noise and artifacts and it is essential to remove the noise in order to support any decision making for specialist. It is very difficult to remove the noise from 12 channel ECG waveforms using standard noise removal methodologies. Removal of the noise from ECG waveforms is majorly classified into two types in signal processing namely Digital filters and Analog filters. Digital filters are more accurate than analog filters because analog filters introduce nonlinear phase shift. Most advanced research digital filters are FIR and IIR.FIR filters are stable as they have non-recursive structure. They give the exact linear phase and efficiently realizable in hardware. The filter response is finite duration. Thus noise removal using FIR digital filter is better option in comparison with IIR digital filter. But it is very difficult to find the cut-off frequency parameter for dynamic multi-channel ECG waveforms using existing traditional methods.
\end{abstract}

So, in this research, newly introduced Multi-Swarm Optimization (MSO) methodology for automatically identifying the cut-off frequency parameter of multichannel ECG waveforms for low-pass filtering is inspecting. Generally, the spectrums of the ECG waveforms are extracted from four classes: normal sinus rhythm, atria fibrillation, arrhythmia and supraventricular. Baseline wander is removed using the Moving Median Filter. A dataset of the extracted features of the ECG spectrums is used to train the MSO. The performance of the MSO with various parameters is investigated. Finally, the MSO-identified cut-off frequency parameter, it's applied to a Finite Impulse Response (FIR) filter. The resulting signal is evaluated against the original clean and conventional filtered ECG signal.

Keywords: 12 Channel ECG Waveforms, Multi Swarm Optimization Neural Network, Low-pass filtering, Finite Impulse Response (FIR).

\section{INTRODUCTION}

The measurement of the electrical activity of the heart (cardiac) muscle is called electrocardiogram (ECG). As the heart performs its function of pumping blood through the circulatory system, the result of the mechanical events within the heart is the generation of a certain sequence of electrical events [1]. The ECG waveform consists of $\mathrm{P}$ wave, QRS wave, T wave and $\mathrm{U}$ Wave. Basic unit of the ECG waveform is shown below figure 1.

Nowadays, most of the health care and cardio centres are using the most advanced ECG equipment like 12 Channel/Lead ECG. The 12-Channel ECG provides the most thorough ability to interpret electrical activity within the heart compare with standard ECG Waveforms. In a 12-Channel ECG, an electrode is placed on each upper arm and lower leg to monitor the standard Channels (I, II, and III) and augmented leads (aVR, aVL, and aVF) along the frontal plane. In addition, chest leads may be used to evaluate the horizontal plane of electrical activity through assessment of V1 to V6. Most ECG devices are configured with computer systems and analysis the ECG waveforms. 12 channel ECG waveforms are shown below figure2.

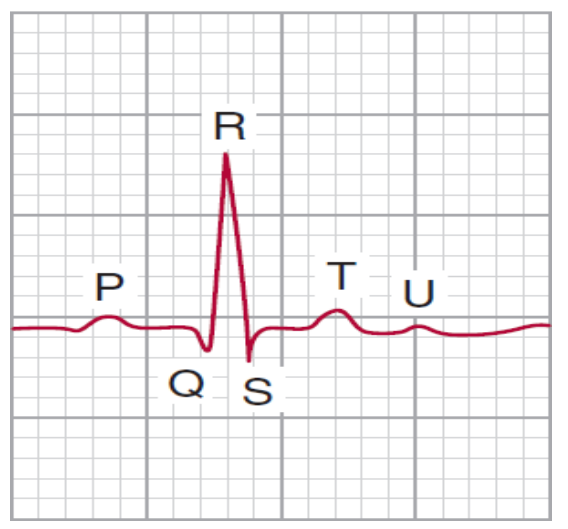

Fig 1 Basic unit of the ECG waveform

Figure 2 shows 4 rows and each row contains 3 channels. For computerized interpretation system and for human electro 
cardiographer, it is vital to access accurate measurements of ECG intervals and critical points (e.g. P, QRS, and T). Basic intervals are showing below figure 3 .

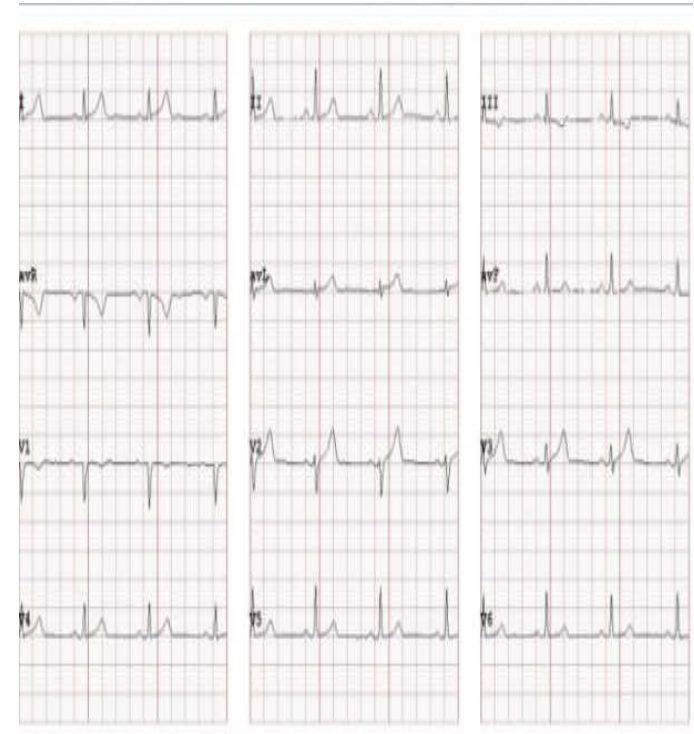

Fig 2 12-Channel ECG waveform

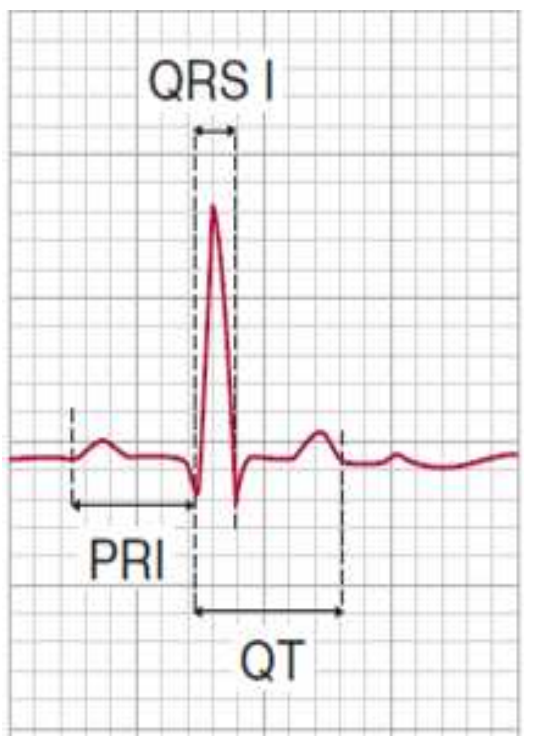

Fig 3 Basic intervals

The morphology of ECG waveform has been used for recognizing heart activity. Therefore, it is very important to obtain the parameters of clear ECG waveforms without noise [2-3]. ECG provides valuable information to diagnose heart disorders and the ischemic changes that may occur, such as the myocardial infarction, conduction defects and arrhythmia [3]. Therefore, the ECG waveform must be clearly represented and filtered to remove all distracting noise and artifacts.
The ECG waveform is usually corrupted with noise from various sources including imperfect contact of electrodes to the body, machine malfunction and electrical noise from elsewhere in the body, respiration and muscle contractions [5]. The produced noise consists of low-frequency components that cause baseline wander and high-frequency components such as power-line interference [3]. ECG noise removal is complicated due to the time varying nature of ECG waveforms. In noise removal, it is necessary to identify the cut-off frequency parameter of the filter. However, this is difficult to determine and improper treatment may introduce additional artifacts to the signal especially on the QRS wave.

Although there are many new methods for noise removal, most noise removal systems for ECG waveforms use the band pass filter because of simplicity in implementation and requiring minimal coefficients [2-6]. The important consideration of this popular technique is in automatically identifying the correct pass bands in the frequency spectrum using the intelligent methods. The intelligent methods are Artificial Neural Networks (ANN), Swarm Intelligence (SI) and Support Vector Machine (SVM) etc. The next section provides a brief review on some recent noise removal methods [7].

Analog filters can also be used to remove these noises, but nonlinear phase shift is introduced by them. Digital filters are more accurate and precise than analog filters. Digital filters are of two kinds- one is Finite Impulse Response (FIR) and second one is Infinite Impulse Response (IIR). The FIR filters have more advantages over IIR filters.

An insufficient and incomplete research effort has been observed in the area of automated calculation of cut-off frequency parameters for noise removal using filters. Therefore, the incompleteness in this area has been the motivation factor to pursue the present research i.e. to find cut-off frequency parameter using FIR filter with MSO methodology. Earlier application of PSO in training the ANN to identify the optimal cut-off frequency parameter for removal of the high frequency noise in ECG signals with FIR filter is used. In this study, FIR filter is preferred to compare with IIR filter and is more susceptible to problems of finite-length arithmetic [7-10]. Additionally, FIR filters are less complex and can be readily applied to ECG waveforms. Above mentioned knowledge is not sufficient for noise removal in multi-channel ECG waveforms. So this paper proposes the new methodology for removal of noise in multi-channel ECG waveform. One of the weaknesses of regular particle swarm optimization (PSO) is that results depend to a large extent on the starting position of the particles. Multi-swarm optimization (MSO) uses several swarms of particles rather than a single swarm. A collection of particles is called a swarm. This approach helps overcome the sensitivity to initial positions. MSO is very robust and advanced optimization algorithm's is an extension of PSO. The position of each virtual particle represents a potential solution and particles iteratively 
move to better positions until some stopping condition is met [15-19].

\section{BACK GROUND}

Noise removal from ECG waveforms has been discussed so many research workers. Most of the research works are ECG waveform removed noise only for one channel waveform. Kang-Ming Chang proposed on Ensemble Empirical Mode decomposition for noise removal process for multi-channel ECG waveforms. In (2013), Principal Component Regression is used for remove the approach for maternal ECG removal and multichannel correlation based fHR detector. Zhang and Sui [14] proposed a method based on morphological filtering and wavelets to eliminate the noise in ECG waveforms and increase the diagnosis efficiency.

In [15], intelligent approach based on moving median filter and Self-Organizing Map (SOM) neural network is proposed to identify the cut-off frequency parameter of the noise, which is to be filtered out and proved results of the proposed scheme are compared with the low-pass FIR filtering for ECG signal high frequency noise removal. Poungponsri and Yu (2009) used the Wavelet Neural Network (WNN) for ECG waveform modelling and noise reduction. The WNN built combined the multi resolution nature of wavelets and the adaptive learning ability of ANN, and was trained by a hybrid algorithm that includes the Adaptive Diversity Learning Particle Swarm Optimization (ADLPSO) and the gradient descent optimization.

Previous studies have indicated that the neural network based methods present effective approaches for denoising ECG waveforms. There are also some algorithms for optimization of the ANN itself [7]. And still more need to improve the performance for multi-channel in dynamic environments.

So, the most recent swarm optimization algorithm is the MultiSwarm optimization (MSO) algorithm. This MSO algorithm is randomly divides initialized particles into several positions. It's identify the cut-off frequency parameter with calculating certain generations respectively, every position is combined into one position and continues to calculate until the stop condition is satisfied. At the same time, the MSO updates the positions by following multi-gbest and multi-pbest instead of single gbest and single pbest [18-19].

With regards to filters for noise removal, there is insufficient research in the area of automated calculation of cut-off frequency parameter for multi-channel Waveforms. This paper presents application of MSO in training the ANN to identify the optimal cut-off frequency parameter for removal of high frequency noise in ECG signals using an FIR filter. Additionally, FIR filters are less complex and can be readily applied to ECG waveforms.

\section{PROPOSED METHODOLOGY}

Proposed methodology is shown below figure 4 .

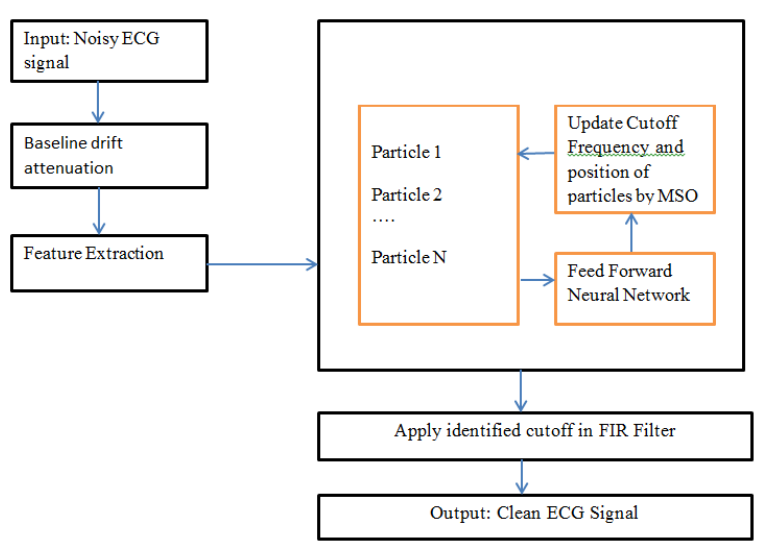

Fig 4 Flow chart of proposed methodology architecture

Major process divided into four steps:

- The initial step is data preparation, whose details are explained in the subsequent sections.

- Next followed step is drift noise removal and Feature Extraction.

- The heart Step for this research is MSO system configuration, multiple swarms applied to different MSO positions. It will produce the Cutoff frequency parameter for filter methods.

- Final Step is applying the cutoff frequency parameter value in FIR filter method. It gives clean ECG signal without noise for analysis of ECG further levels.

\subsection{Test Data}

In this Research, data is collected from three centers:

PTB Diagnostic ECG Database: This database contains 549 records from 290 subjects. Each subject is represented by one to five records. Each record includes 15 simultaneously measured signals. Each signal is digitized at 1000 samples per second, with 16 bit resolution over a range of $\pm 16.384 \mathrm{mV}$.

St.-Petersburg Institute of Cardio logical Technics 12-lead Arrhythmia Database: This database contains 75 twelve-lead ECGs from 32 Holter records. Each record is 30 minutes long and contains 12 standard leads, each sampled at $257 \mathrm{~Hz}$, with gains varying from 250 to 1100 analog-to-digital converter units per millivolt.

PhysioNet/Computing in Cardiology Challenge 2011: This database contains 1500 twelve-lead ECGs. These ECGs have been classified individually with respect to acceptability for purposes of diagnostic interpretation. One sample data set from test data of the 12 channel ECG waveforms is shown below figure 5 . 


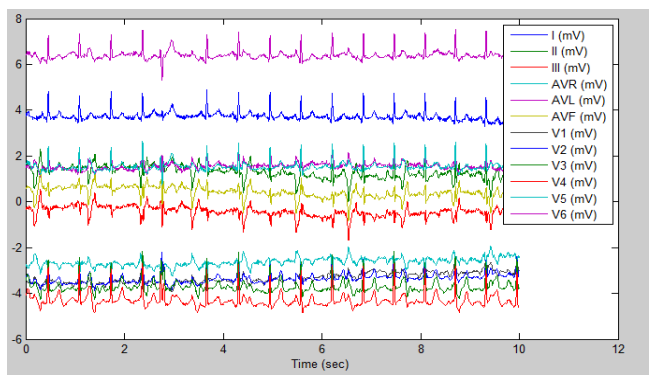

Fig 5 Sample Data set from test data of the 12 Channel ECG waveforms

\subsection{Removal of Baseline Wander}

Baseline wander makes manual and automatic analysis of 12 channel ECG records is very hard, especially in measuring of the ST segment deviation that is used for diagnostic ischemia. Baseline wander removal is a filtering need which can be implemented efficiently in an embedded platform. However, inaccurate baseline wander removal can cause distortion of important clinical information, particularly ST segment distortion, as there are overlaps in the spectrum of the baseline wander and low frequency components of the ECG signals.

One of the 12 Channels ECG waveforms for baseline wander removal is presented in below Figure 6. As such, the first step is the reduction improvement for the removal of baseline wander. In part of the reduction, select each channel as input of the filter .One of the channels from Figure 5 is shown in Figure 6 after applying the following equation (1). As part of the analysis, median filter is known to be more proficient and it is applied in this work.

$$
\text { B1 = Median Filter(ON.mat, [a b]) ---- (1) }
$$

Each output data point contains the median value in the [a b] subpart of the corresponding data in the input ECG waveforms. Same equation (1) implemented for all left 11 channels in part of the 12 channel ECG waveforms. Finally, the baseline wander is eliminated successfully and producing a virtually zero attenuation of the ECG waveform as shown below figure 7 for one sample channel from the test data sets.

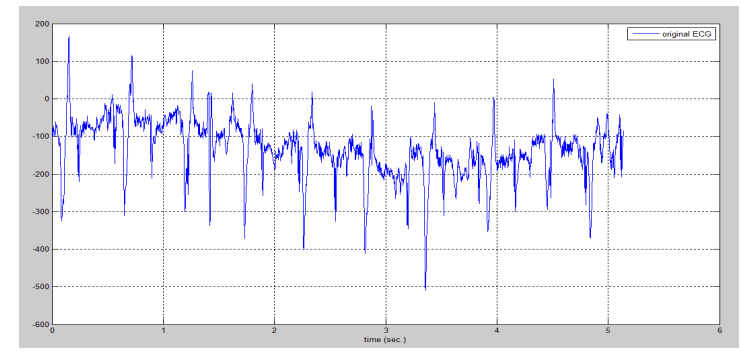

Fig 6 III Channel ECG waveform from the sample of 12 Channel ECG waveforms

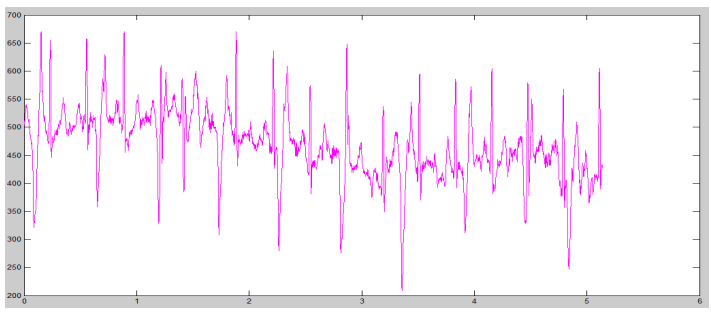

Fig 7: Median filter for figure 6

\subsection{Feature Extraction}

MSO needs some inputs - derived from 22 features of the test data sets. Analysis of the ECG waveform, taking into account minimal complexity in computation, revealed that two common statistical measures namely standard deviation and variance would suffice. MSO methodology needs more inputs for each data set point and in this paper it is included new interval feature compare with existing research scope. In addition, some of the standard attributes like mean and median are considered. Another additional feature is Form Factor (FF). Form factor (FF) is another technique to represent ECG waveform complexity in a scalar value. All above features are used to find the cut-off frequency using MSO methodology. List of the features is shown below table 1 .

Table 1 Extract features descriptions

\begin{tabular}{|l|l|l|l|}
\hline $\begin{array}{l}\text { Feature } \\
\text { No. }\end{array}$ & $\begin{array}{l}\text { Descript } \\
\text { ion }\end{array}$ & $\begin{array}{l}\text { Featur } \\
\text { e No. }\end{array}$ & Description \\
\hline 1 & $\mathrm{X}(\mathrm{R} 1)$ & 13 & $\mathrm{X}(\mathrm{R} 2)$ \\
\hline 2 & $\mathrm{~V}(\mathrm{R} 1)$ & 14 & $\mathrm{~V}(\mathrm{R} 2)$ \\
\hline 3 & $\mathrm{X}(\mathrm{S})$ & 15 & $\mathrm{X}(\mathrm{R} 2)-\mathrm{X}(\mathrm{R} 1)$ \\
\hline 4 & $\mathrm{~V}(\mathrm{~S})$ & 16 & $\mathrm{~V}(\mathrm{R} 2)-\mathrm{V}(\mathrm{R} 1)$ \\
\hline 5 & $\mathrm{X}(\mathrm{T})$ & 17 & $\mathrm{X}(\mathrm{S})-\mathrm{X}(\mathrm{R} 1)$ \\
\hline 6 & $\mathrm{~V}(\mathrm{~T})$ & 18 & $\mathrm{X}(\mathrm{T})-\mathrm{X}(\mathrm{S})$ \\
\hline 7 & $\mathrm{X}(\mathrm{P})$ & 19 & $\mathrm{X}(\mathrm{P})-\mathrm{X}(\mathrm{T})$ \\
\hline 8 & $\mathrm{~V}(\mathrm{P})$ & 20 & $\mathrm{X}(\mathrm{Q})-\mathrm{X}(\mathrm{P})$ \\
\hline 9 & $\mathrm{X}(\mathrm{Q})$ & 21 & $\mathrm{X}(\mathrm{R} 2)-\mathrm{X}(\mathrm{Q})$ \\
\hline 10 & $\mathrm{~V}(\mathrm{Q})$ & 22 & Median \\
\hline 11 & $\mathrm{SD}$ & 23 & Form Factor \\
\hline 12 & Mean & 24 & Average of intervals \\
\hline
\end{tabular}

\subsection{Multi Swarm Optimization (MSO)}

MSO is a technique for estimating the solution to difficult or impossible numerical problems. It is an alternative of particle swarm optimization (PSO) based on the use of multiple subswarms instead of one (standard) swarm. The general approach in multi-swarm optimization is that each sub-swarm focuses on a specific region while a specific diversification method decides where and when to launch the sub-swarms. The multiswarm framework is especially fitted for the optimization on multi-modal problems. High level algorithm for MSO is shown in figure 8. 


\begin{tabular}{|l|}
\hline Algorithm Multi-swarm optimization () \\
\{ \\
loop maxLoop times \\
for each swarm \\
for each particle \\
compute new cutoff frequency \\
use cutoff frequency to update position \\
check if a new best cutoff frequency has been \\
found \\
end for \\
end for \\
end loop \\
\} \\
\hline
\end{tabular}

Fig 8: High level algorithm for MSO optimization

The particles or inputs of the Multi-swarm optimization are determined by the features of the dataset. MSO Particles bases its diversification mechanism on the "collision" of particles. When particles get too close, a repulsive force expels the particles into new waves/sub-swarms, and this avoids a complete convergence. A key feature of the new sub-swarms is that their initial positions are not randomly selected as in normal swarms. Instead, they maintain some information from the previous trajectories of the particles. A similar relationship exists with initial velocities.

This multiswarm system bases its diversification mechanism on a "devour and move on" strategy. Once a sub-swarm has devoured a region (intensive search) the swarm is ready to move on to another promising region. The initial positions of the new sub-swarm are selected using a scouting process around the best position found by the previous sub-swarm. With fewer iterations per particle, it may be beneficial to increase the convergence rate of the sub-swarms (i.e. decrease the constriction factor). In standard PSO the velocities of each particle are updated by

$$
v_{d}=x\left(v_{d}+c_{1} \in \underset{1}{{ }_{1}} \underset{x_{d}^{-----(2)}}{\text { pbest } \left._{d}-x_{d}\right)+c_{2}} \in{ }_{2} \text { (gbest }_{d}-\right.
$$

In (2), $\mathrm{v}$ is the particle's velocity; $\mathrm{x}$ is the position of the particle, and $d$ isa given dimension. The variables $\in 1$ and $\in$ 2 are random values, which to gether with the weights c1 and c2 determine the contribution of attractions to the personal and global bests pbestd and gbestd respectively. The constriction factor is represented by $\mathrm{x}$, the specific value used for the constriction factor in [6] is $x=0.792$. By changing the value of this parameter, it is possible to modify the particle's momentum, and therefore either promote a more exploratory or amore exploitative behaviour.

\subsection{Finite Impulse Response Filter with MSO}

The impulse response of an Nth-order discrete-time FIR filter lasts for $\mathrm{N}+1$ samples, and then settles to zero. Higher orders give sharper cut-off in the frequency response therefore; the desired sharpness will determine the filtering order. The default window is the Hamming of size $\mathrm{N}+1$. The FIR filter (w) is represented by

$$
w=0.54-0.46 \cos \left(\frac{2 \pi n}{N}\right) \text { for } 0 \leq \mathrm{n} \leq \mathrm{N}---(3)
$$

The cut-off frequency must be identified for the ECG signal to be filtered. The next section provides the test results achieved for automatic identification of the cut-off frequency by the MSONN and the FIR filter in denoising the ECG signals.

\section{ANALYSIS OF EXPERIMENTAL}

The proposed system performance using the configured MSO with NN is tested by measuring various parameters. The FIR is implemented with the cut-off frequency parameter identified by the MSO and the results are evaluated against the clean and conventionally filtered 12 channel ECG Waveform. Validate the using k-folding and $\mathrm{m} \mathrm{K}$ Swam method with $\mathrm{k}=3$ to 10 . For each of the $\mathrm{k}$ times of $\mathrm{NN}$ training with different partitions of the training data and test data. One of the NN method is $70 \%$ of the samples in the dataset were used for training with the nonover lapping $30 \%$ for testing.

Clean signals would allow for more accurate evaluation of the effectiveness of the proposed method in terms of identification of appropriate cut-off values. Test data sets collected from PhysioNet data center with original that are influenced by noise, and there is no access to actual clean signals. Some of the noise signals are simulated by adding a small value of high frequency components of random noise mixed the natural noise. In this paper, two methods are used for analyzing the noise accuracy namely Mean Squared Error and another one is Membership Functions. The Mean Squared Error (MSE) is measured for varying numbers of hidden nodes and number of training points. To decrease the complexity of the network and increase training speed, fewer nodes in the hidden layer are preferred. The MSE equation is shown below (4):

$$
M S E=\frac{1}{N} \sum_{n=0}^{N-1}\left[y_{n}-t_{n}\right]^{2}
$$

Where $y_{\eta}$ is the output of the network, $t_{n}$ is the desired target and $N$ is the number of test records. Membership Functions for each input and output, training data sets, type of MFs for each input and output, MSONN and etc. is determined by learning data. The MFs change after the training according to the level of noise present in signal. Increasing number of training epochs decreases Mean Square Error (MSE) but after 100, 500training samples MSE achieve steady state level because parameters of MFs are stable. First case research start with some assumed value with FIR. 


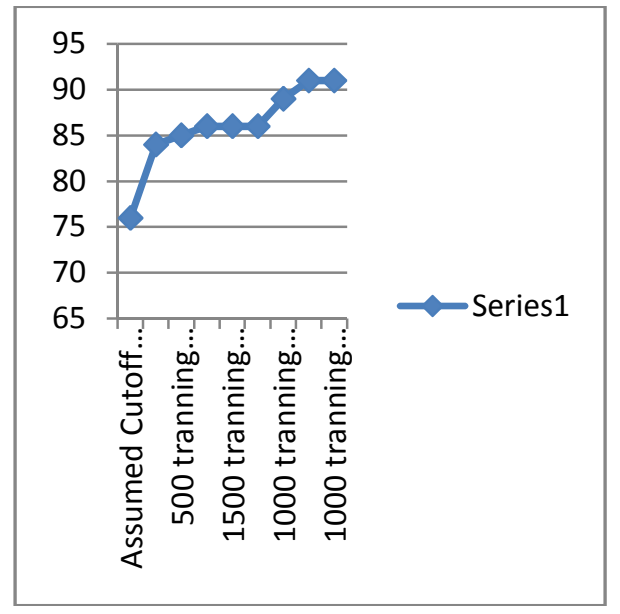

Fig 9: Performance analysis accuracy results for FIR with MSO $\mathrm{NN}$

Then this research is used the MSO NN with 100, 500, 1000, 1500 and 2000 training samples with 3 nodes of the NN and opposite direction identical increased the test data ratio also, symbolized in above figure 9 . Outcome of research is each time performance is increased when sample increased after 1000 samples, it shows same accuracy. So changed the testing point of view of this research is start the increasing of the NN node with hidden layers, then outcome this direction of the research give some more better results. But in this direction after 7 hidden layers, gives same accuracy performance. Figure 9 shows performance the MSE results achieved by using different ways of the research outcomes. It shows that the MSONN has the best performance with 7 nodes in the hidden layer with 1000 training samples with test data.

\section{CONCLUSIONS}

In this paper, proposed a novel method to find the cut-off frequency parameter using MSO is used to remove noise in 12 channel ECG waveforms. Multi-swarm generalizations of particle swarms and compared their performance on a benchmark dynamic multi-modal environment and parallel for each channel using different sub swarms. It is an assembly of charged particle swarm optimizers. It is already known that surrounding a neutral or conventional PSO sub-swarm with an orbiting sub-swarm of mutually repelling particles increases swarm diversity. This improves performance of the multichannel Waveforms cut-off frequency identification in terms of SNR and time complexity. Compared different NN hidden layer gives best results using 10 Swarm and 2 hidden layers.

Future extended problem with more sub-swarms with increase of the multilevel hidden layers to find the different cut-off frequency parameters.

\section{REFERENCES}

[1]. Jenkins,Peggy, "Nurse to Nurse ECG Interpretation" McGraw-Hill Professional, Published in 2009

[2]. Bhumika Chandrakar1, O.P.Yadav2, V.K.Chandra, " $A$ Survey Of Noise Removal Techniques For ECG Signals" International Journal of Advanced Research in Computer and Communication Engineering Vol. 2, Issue 3, March 2013, pp, 1354-1357

[3]. Jukka A Lipponen, Mika P Tarvainen "Advanced Maternal ECG Removal and Noise Reduction for Application of Fetal QRS Detection" Computing in Cardiology 2013 PP. 161-164.

[4]. ManpreetKaur, Birmohan Singh, Seema "Comparisons of Different Approaches for Removal ofBaseline Wander from ECG Sign" International Journal of Computer Applications 2011 pp, 32-36

[5]. V'ictorBarbero Romero, Profesor director: David Atienza Alonso, Profesoracolaboradora: Nadia Khaled, $\mathrm{UC} 3 \mathrm{M}$, "ECG baseline wander removal and noise suppression analysis in an embedded platform" International Conference of Curso 2008-2009.

[6]. Sara Moein, Rajasvaran Logeswaran and Khazaimatol Shima Subari "An Automated Intelligent Approach for ECG Signal Noise Removal" International Journal of Computer Science Issues 2012.

[7]. Hassoun M.H., "Fundamentals of Artificial Neural Network", MIT 1995.

[8]. Minami K., Nakajima H., Toyoshima T., "Real-time discrimination of ventricular tachyarrhythmia withFourier transform neural network", IEEE, 1999

[9]. Jalal A. Nasiri, Mahmoud Naghibzadeh," $E C G$ Arrhythmia Classification with Support Vector Machines and Genetic Algorithm" IEEE 2009 Third UKSim European Symposium on Computer Modeling and Simulation pp 187-192.

[10]. K.D. CHINCHKHEDE,GOVIND SHARAN YADAV, S.R HIREKHAN, D.R SOLANKE, "On the Implementation of FIR Filter with Various Windows for Enhancement of ECG signal" IJEST 2011,PP, 20312040.

[11]. FoteiniAgrafioti, DimitriosHatzinakos, 'An Enhanced EMD Algorithm For ECG Signal Processing” Ieee-Dsp 2011 pp, 978-984

[12]. Rui Rodrigues and Paula Couto,"A Neural Network Approach to ECG Denoising" arXiv:1212.5217v1 [cs.CE] 20 Dec 2012

[13]. M. K. Islam, A. N. M. M. Haque, G. Tangim, T. Ahammad, and M. R. H. Khondokar, Member, IACSIT , "Study and Analysis of ECG Signal Using MATLAB \& LABVIEW as Effective Tool" International Journal of Computer and Electrical Engineering, Vol. 4, No. 3, June 2012 , pp , 404-408.

[14]. Kang-Ming Chang "Arrhythmia ECG Noise Reduction by Ensemble Empirical Mode Decomposition” Sensors 2010, 6063-6080. 
[15]. Sara Moein, RajasvaranLogeswaran, "Intelligent ECG Signal Noise Removal using PSONN" International Journal of Computer Applications' (0975 - 8887) Volume 45- No.6, May 2012

[16]. Junliang Li , Xinping Xiao "Multi- Swarm and MultiBest particle swarm optimization algorithm" IEEE 2008 PP $6281-6286$.

[17]. Omran, M. G., Salman, A., \&Engelbrecht, "Particle swarm optimization forpattern recognition and image processing”. Springer 2008.

[18]. Blackwell, T. M., \&Branke, J. "Multi-swarms, exclusion, and anti-convergence in dynamic environments", IEEE Transactions on Evolutionary Computation, 2004 PP 51-58.

[19]. Zhao, Liang.J.J, Suganthan .P.N,"Dynamic Multi-Swarm Particle Swarm Optimizer with Local Search for Large Scale Global Optimization" IEEE Congress on Evolutionary Computation 2008, pp. 3845-3852

\section{BIOGRAPHIES:}

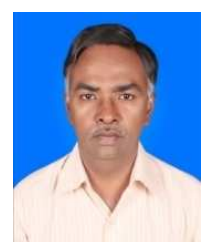

Prof. M.Srinivasulu, received his B.Tech Degree in Electronics \& Communication Engineering and M.Tech degree in Electronic Instrumentation \& Communication Systems from S V University-Tirupati, A.P-India. He is currently working as Professor in the Department of ECE in LAQSHYA INSTITUTE OF TECHNOLOGY \& SCIENCES, Khammam, A.P-India. His research interests on Signal Processing and Communication Systems. He is a Member of IEEE, Member of Institution of Engineers (MIE) and Member of Indian Society for Technical Education (MISTE).

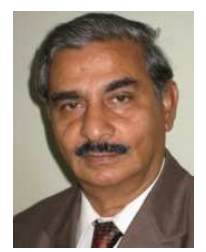

Dr.K.CHENNAKESHAVA REDDY is the Principal, Bharathi Engg. College and Professor of Electronics \& Communication Engg. He did his B.E. \& M.Tech. from Regional Engg. College-Warangal and Ph.D. from JNTU-Hyderabad. He was the former Director of Evolution -JNTUH, Principal of JNTU -Jagtiyal \& Deputy Director of UGC-Academic Staff college -JNTUH.He was an expert committee member constituted by AICTE, South Western region, Bangalore. He Published 20-International, 50-National Research papers in various international \& National Journals. He also guided 16 Ph.D. Research scholars. 